\title{
High Disease Activity Is Associated with Self-reported Depression and Predicts Persistent Depression in Early Rheumatoid Arthritis: Results from the Ontario Best Practices Research Initiative
}

\author{
Bindee Kuriya, Raman Joshi, Mohammad Movahedi, Emmanouil Rampakakis, John S. Sampalis, \\ Claire Bombardier, and Ontario Best Practices Research Initiative Investigators
}

\begin{abstract}
Objective. We sought to determine if initial high disease activity or changes in disease activity contribute to persistent depression in early rheumatoid arthritis (ERA). We also determined if disease activity and depression is modified by sex.

Methods. Depression was ascertained by self-report among patients enrolled in the Ontario Best Practices Research Initiative. The association between baseline disease activity, measured by the Clinical Disease Activity Index (CDAI), and persistent depression was evaluated with multivariate regression models, and effect modification by sex was tested. A general estimating equation assessed the association between change in CDAI over time and risk of depression.

Results. The sample of 469 ERA subjects was predominantly female (73\%). At baseline, the prevalence of depression was $26 \%$, and $23 \%$ reported persistent depression. After adjusting for potential confounders, higher baseline CDAI was associated with both baseline and persistent depression (OR 1.03, 95\% CI 1.01-1.05). Female sex was an effect modifier of this relationship (OR 1.04, 95\% CI 1.01-1.06). Maintaining a moderate or high CDAI score over 2 years also increased the risk of future depression. Conclusion. Depression in ERA is common and initial high disease activity is associated with the probability of depression and its persistence. This risk seems particularly modified in women with active disease and represents an area for targeted focus and screening. Future studies in ERA are needed to determine if intervening during the "window of opportunity" to control disease activity has the potential to mitigate the development and maintenance of adverse mental health outcomes, including depression. (First Release May 15 2018; J Rheumatol 2018;45:1101-8; doi:10.3899/ jrheum.171195)
\end{abstract}

Key Indexing Terms:

DISEASE ACTIVITY

\section{DEPRESSION}

RHEUMATOID ARTHRITIS

\section{PROGNOSIS}

From the Sinai Health System, University of Toronto; Toronto General Hospital Research Institute, University Health Network, University of Toronto, Toronto; Brampton Civic Hospital, William Osler Health System, Brampton, Ontario; JSS Medical Research, St. Laurent; Mc Gill University, Montreal, Quebec, Canada.

Dr. Kuriya was the recipient of the Canadian Rheumatology Association/The Arthritis Society Clinician Investigator Award. OBRI is funded by peerreviewed grants from the Canadian Institute for Health Research (CIHR), Ontario Ministry of Health and Long-Term Care, Canadian Arthritis Network and unrestricted grants from Abbvie, Amgen, Bristol Myers Squibb, Pfizer, UCB, Janssen and Roche, Celgene, Fresenius Kabi.

B. Kuriya, MD, FRCPC, SM, Sinai Health System, University of Toronto; R. Joshi, MD, Brampton Civic Hospital, William Osler Health System; M. Movahedi, MD, PhD, Toronto General Hospital Research Institute, University Health Network, University of Toronto, and JSS Medical Research; E. Rampakakis, PhD, JSS Medical Research, S. Sampalis, PhD, JSS Medical Research, and McGill University; C. Bombardier, MD, FRCPC, MSc, Sinai Health System, University of Toronto, and Toronto General Hospital Research Institute, University Health Network, University of Toronto.

Address correspondence to Dr. B. Kuriya, Division of Rheumatology, Sinai Health System, The Joseph and Wolf Lebovic Building, 60 Murray Street, Room 2-008, Toronto, Ontario M5T 3L9, Canada.

E-mail: bindee.kuriya@sinaihealthsystem.ca

Accepted for publication February 12, 2018.
Rheumatoid arthritis (RA) is a systemic inflammatory disease that has both physical and psychological manifestations and affects up to $1 \%$ of the population worldwide. RA is recognized as a disease of multimorbidity and is associated with the development of other chronic medical conditions, many of which may also be mediated by chronic immune dysregulation ${ }^{1}$. Of these, depression has been cited as the most frequent comorbidity in RA, with lifetime prevalence estimates ranging from $14 \%$ to $48 \%$, depending on the outcome definition used ${ }^{2,3}$.

Depression is a major health concern for individuals living with RA, as well as for those who treat them, because of its link with negative health outcomes, including higher pain, worse disability, reduced medication adherence, reduced therapeutic efficacy, greater health service use, and even risk of death ${ }^{4,5}$.

Depression is often episodic and may present across the spectrum of RA disease duration. Of interest, studies have demonstrated an elevated risk of depression during the asymptomatic but immunologically active phase of disease

Personal non-commercial use only. The Journal of Rheumatology Copyright @ 2018 . All rights reserved. 
(pre-RA), and a substantially higher risk during the initial years of clinically apparent disease $e^{6,7,8}$. This raises the question of whether systemic inflammatory pathways simultaneously cause the articular symptoms of RA and trigger depression, or whether the response to inflammation (e.g., pain, coping, medications) is the key driver of psychological manifestations.

There have been many attempts to identify which factors may predict the development of depression in RA to facilitate comprehensive treatment, and ideally, preventive approaches. Studies have consistently identified low socioeconomic status, female sex, age, ethnicity, pain, and functional limitation to be associated with a greater risk of depression ${ }^{4}$.

The extent to which active disease contributes to or maintains depression has not been well studied. Specifically, few studies are available on this topic in early RA (ERA) and have limitations. In a previous paper, Kekow, et al demonstrated that clinical remission, according to the 28 -joint count Disease Activity Score (DAS28), significantly reduced symptoms of depression/anxiety compared to those who did not achieve remission over a 2-year period; these were data from a clinical trial with patient characteristics not generalizable to routine clinical practice ${ }^{9}$. Another study found no association between disease activity and depression or severity of depressive symptoms. This was primarily limited by the cross-sectional design, preventing any conclusions about the temporal relationship between disease activity and depression ${ }^{10}$. Overman, et al attempted to study the prospective association between psychological distress and disease activity in ERA ${ }^{11}$. They concluded that a higher level of disease activity is a risk factor for increased psychological distress; however, the outcome was only measured at baseline and 6 months later ${ }^{11}$. In contrast, a longer study by Bacconnier, et al noted a decreasing prevalence of psychological distress over 3 years among patients with very early RA (symptom duration $<6$ mos), but found no significant association between sex, inflammatory variables, or treatment options and risk of psychological symptoms ${ }^{12}$. Increasing DAS28 values using C-reactive protein (DAS28-CRP) were associated with self-reported distress at 3 years, but not consistently over followup ${ }^{12}$.

Taken together, there are conflicting reports on whether active disease, especially in ERA, represents a potential modifiable risk factor to prevent or reduce the severity of depression. Moreover, it is not known if initial high disease activity (HDA; or which of its components) contributes to persistent depressive symptoms. We attempted to fill these knowledge gaps and test our hypothesis that initial HDA would increase the risk for persistent depression. Since the prevalence of depression in ERA is reported to be higher in females, we additionally sought to determine if the effect of disease activity on persistent depression differed between males and females. Last, we evaluated how changes in disease activity over time influence the future risk of depression among individuals with ERA followed in routine care.

\section{MATERIALS AND METHODS}

Data source and patients. The Ontario Best Practices Research Initiative (OBRI) is a provincial prospective registry of patients with RA that gathers longterm information on clinical practice patterns of patients with RA followed in routine care. It incorporates physician assessments and a unique method of collecting data from the patients directly using telephone interviewers. Patients are included if they are $\geq 18$ years of age with a physician-confirmed RA diagnosis and $\geq 1$ swollen joint. Patients are recruited at any stage of disease and are treated and followed at the discretion of their rheumatologist.

Between January 2008 and January 2016, 3020 eligible patients across 65 sites gave their consent to participate in physician evaluations, and 2942 agreed to patient interviews.

For our study, we restricted our population to patients with ERA (symptom duration $\leq 1 \mathrm{yr}$ ) with at least 2 years of followup. To obtain the most accurate information from patient interviews and from physician assessments, we excluded patients with a gap $\geq 60$ days between patient enrollment and their first interview. We also applied a further exclusion criterion if patients had $<4$ interview visits over 2 years of followup (Figure 1).

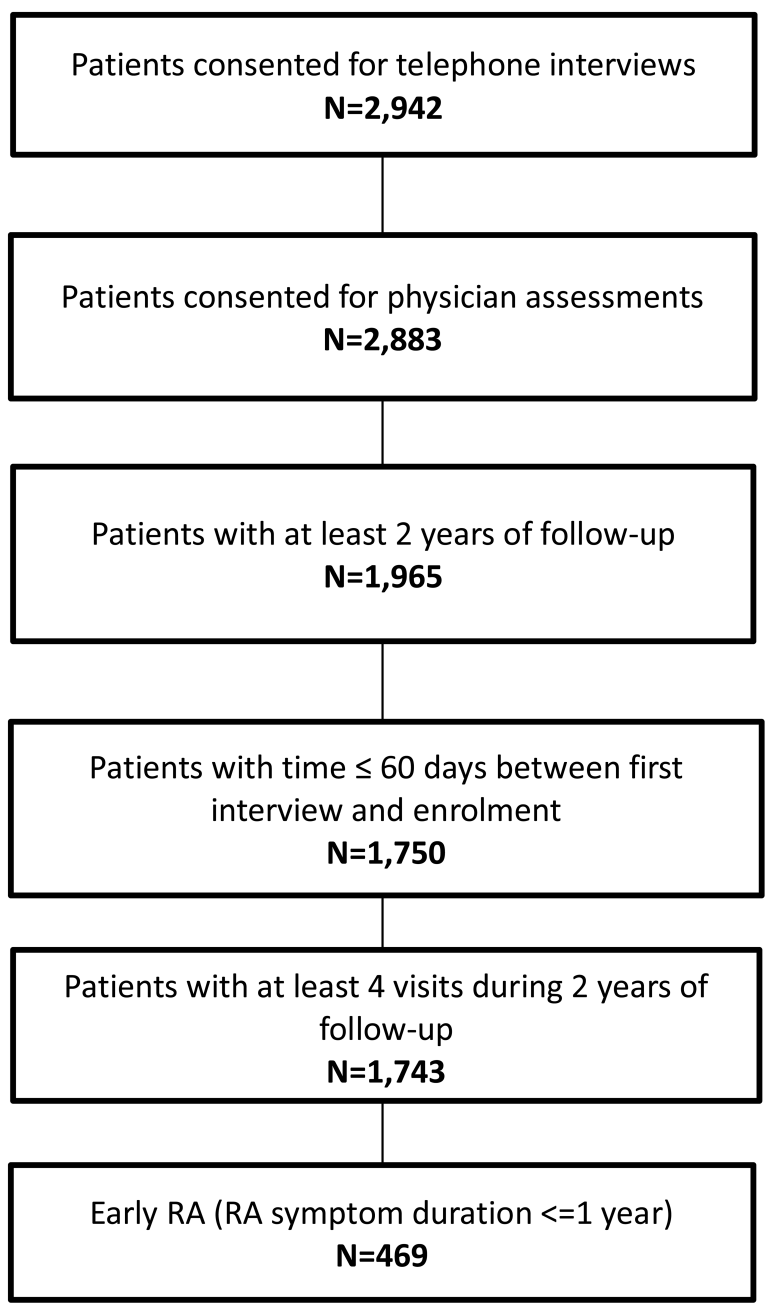

Figure 1. OBRI participant flow diagram. OBRI: Ontario Best Practices Research Initiative; RA: rheumatoid arthritis. 
The University Health Network (UHN) ethics approval was obtained (REB no. 07-0729 AE) for participating investigators working at UHN and for the OBRI data management center, which is located at UHN. We also obtained approval from each of the individual research ethics boards of all institutions before recruitment: Institutional Review Board Services of Mount Sinai Hospital, St. Joseph's Healthcare Hamilton of University of Western Ontario, St. Joseph's Healthcare Toronto, Homewood (Headwaters Health Care Centre), Sault Area Hospital (Group Health Centre), St. Michael's Hospital, Ottawa Hospital, William Osler Health System, and Sunnybrook Health Sciences Centre.

Clinical assessments. The following clinical data were obtained at physician visits: patient's global assessment (PtGA), physician's global assessment (PGA), 28-joint tender joint count (TJC28), 28-joint swollen joint count (SJC28), DAS28 using erythrocyte sedimentation rate (DAS28-ESR), Clinical Disease Activity Index (CDAI), ESR $(\mathrm{mm} / \mathrm{h}), \mathrm{C}$-reactive protein (CRP, mg/l), Health Assessment Questionnaire-Disability Index (HAQ-DI), rheumatoid factor (RF) status, presence of erosive disease, comorbidity profile (heart disease, hypertension, lung disease, diabetes mellitus, gastrointestinal disease, kidney disease, blood disorders and anemia, depression, cancer, osteoporosis, and back pain), and medication use such as disease conventional synthetic disease-modifying antirheumatic drugs (csDMARD) and biologic DMARD (bDMARD).

CDAI score was the disease activity measure of interest. CDAI was considered both on a continuous and categorical scale. CDAI categories were defined as the following: remission (CDAI $\leq 2.8)$, low disease activity (LDA; $2.8<\mathrm{CDAI} \leq 10$ ), moderate disease activity (MDA; $10<\mathrm{CDAI}$ $\leq 22$ ), or HDA (CDAI $>22$ ). In addition, a binary variable of CDAI was defined as remission and/or LDA versus MDA and/or HDA.

Depression definition. At each telephone interview, a standardized questionnaire asked patients to report any comorbidity, including depression, and relevant treatment they may have received or are currently receiving. Depression was defined as self-reported depression (yes/no) at enrollment (herein referred to as baseline), and persistent depression was defined as self-reported depression at baseline and $\geq 50 \%$ of visits over the first 2 years of followup.

Covariates. The following sociodemographic variables were considered as potential explanatory variables of symptomatic depression: age, sex, marital status, education level, annual income, health insurance type, ethnicity, residential area (urban vs rural), HAQ score, HAQ pain $(0-10 \mathrm{~cm}$ on visual analog score; VAS), fatigue ( $0-10 \mathrm{~cm}$ on VAS), RF status, ESR and CRP values, and number of comorbidities other than depression. Treatment variables were also considered: prior and current use of csDMARD and bDMARD, administration route of bDMARD, and current use and dose of oral corticosteroids.

Statistical analysis. Patient characteristics, disease variables, and treatment information at baseline were described using means $( \pm$ SD) for continuous variables, or $\mathrm{n}(\%)$ for categorical variables.

The primary analysis examined the association between disease activity (CDAI as continuous variable) and persistent symptomatic depression using univariate and multivariate logistic regression models. In the multivariate analysis, the association between CDAI and persistent symptomatic depression was adjusted for those explanatory covariates that were significantly associated with persistent depression in the univariate analysis $(\mathrm{p}<0.05)$. In addition, the association between HDA and persistent symptomatic depression was also assessed using CDAI as a categorical or binary variable.

A secondary analysis was conducted to determine which component of the CDAI may drive any association between disease activity and persistent depression. Here, each component of the CDAI (TJC28, SJC28, PtGA, and PGA) at baseline was evaluated for an association with persistent symptomatic depression using multivariate logistic regression and backward stepwise covariate selection $(\mathrm{p}<0.05)$. In this analysis, the interaction between CDAI components was also evaluated.

For the multivariate models described above, the interaction between sex and CDAI was evaluated and, if there was a significant effect modification $(p<0.05)$, the results were also presented for each sex separately.

The tertiary analysis examined the association between CDAI and depression reported over time during the first 2 years of followup using generalized estimating equation (GEE) models. In this analysis, clinical and patient-reported outcomes, as well as treatments, were considered as time-dependent variables. In the multivariate analysis, the association between CDAI and symptomatic depression was adjusted for other explanatory covariates showing a p value $<0.05$ in the univariate analysis.

Sensitivity analysis. The association between baseline CDAI and symptomatic depression over the first 2 years of followup was also examined using patient-reported treatment for depression as the outcome definition, instead of patient-reported symptomatic depression.

All statistical analyses were performed using SAS 9.4 (SAS Institute Inc.).

\section{RESULTS}

Baseline characteristics. The final analytic sample included 469 OBRI participants with ERA. A detailed participant flow diagram is presented as Figure 1. Patient characteristics at the time of OBRI enrollment are summarized in Table 1. Mean age $( \pm \mathrm{SD})$ was $56.8( \pm 13.6)$ years, the majority of patients were female $(73 \%)$, and mean disease duration was 0.4 $( \pm 0.5)$ years. Mean CDAI score reflected HDA of 22.9 $( \pm 14.1)$ and patients reported high disability according to mean HAQ-DI score of $1.1( \pm 0.74)$.

Prevalence of baseline depression and persistent symptomatic depression. At baseline, 120 (26\%) patients self-reported depression. Patients who reported depression were more likely to be female ( $84 \%$ vs $69 \%, p<0.001)$, and have greater mean number of comorbidities than those who did not report depression (4.1 vs 2.7 , respectively, $\mathrm{p}<0.0001$; Supplementary Table 1, available with the online version of this article). Subjects with baseline depression also differed significantly with higher disease activity scores, core components (TJC28, PtGA), and disability scores (Supplementary Table 1). Depression was substantially higher among patients with initial moderate or high CDAI score (87\%). Treatment patterns seemed reflective of this among subjects reporting depression compared to those with no reported depression, with greater use of previous csDMARD (68\% vs $51 \%$, respectively, $\mathrm{p}<0.01)$ and current use of bDMARD $(11 \%$ vs $5 \%$, respectively, $\mathrm{p}<0.02$; Supplementary Table 1 ).

Over the 2 years of followup, the proportion of the total ERA cohort who reported persistent depression was $23 \%$ $(n=108)$. Persistent depression remained significantly higher among patients with initial moderate CDAI (19.3\%) or high CDAI scores (29.2\%) compared to those in CDAI remission or LDA at baseline (14.3\%, $\mathrm{p}=0.02$; data not shown).

Association between baseline disease activity and persistent depression. Table 2 summarizes the results of the primary analysis assessing the association between baseline CDAI and persistent depression. There was a significant association between high continuous CDAI and persistent depression in univariate analysis (OR 1.03, 95\% CI 1.01-1.04). After adjusting for potential confounders, higher initial CDAI

Personal non-commercial use only. The Journal of Rheumatology Copyright (C) 2018. All rights reserved. 
Table 1. Characteristics of OBRI participants at the time of enrolment. Values are mean $\pm \mathrm{SD}$ or $\mathrm{n}(\%)$ unless otherwise specified.

\begin{tabular}{|c|c|}
\hline Characteristics & $\mathrm{n}=469$ \\
\hline \multicolumn{2}{|l|}{ Sociodemographic characteristics } \\
\hline Age, yrs & $56.8 \pm 13.6$ \\
\hline Female & $342(73)$ \\
\hline White & $408(87)$ \\
\hline Married & $337(72)$ \\
\hline Postsecondary education & $273(58)$ \\
\hline Household annual income, $\geq$ Can $\$ 50,000$ & $233(50)$ \\
\hline Private insurance & $353(75)$ \\
\hline Urban residence & $394(84)$ \\
\hline \multicolumn{2}{|l|}{ Disease characteristics } \\
\hline Disease duration, yrs & $0.4 \pm 0.5$ \\
\hline SJC28, range $0-28, \mathrm{n}=452$ & $5.9 \pm 5.2$ \\
\hline TJC28, range $0-28, n=448$ & $7.2 \pm 6.7$ \\
\hline PtGA, range $0-10, n=418$ & $4.9 \pm 2.7$ \\
\hline PGA, range $0-10, n=412$ & $4.7 \pm 2.3$ \\
\hline CDAI, range $0-76, n=421$ & $22.9 \pm 14.1$ \\
\hline HAQ-DI, range $0-3, n=467$ & $1.1 \pm 0.74$ \\
\hline HAQ pain index, range $0-3, n=467$ & $1.4 \pm 0.85$ \\
\hline Fatigue score, range $0-10, n=467$ & $4.7 \pm 3.1$ \\
\hline $\mathrm{ESR}, \mathrm{mm} / \mathrm{h}, \mathrm{n}=419$ & $25.8 \pm 21.6$ \\
\hline $\mathrm{CRP}, \mathrm{mg} / \mathrm{l}, \mathrm{n}=372$ & $14.8 \pm 23.0$ \\
\hline RF-positive & $293(63)$ \\
\hline Presence of erosions & $179(38)$ \\
\hline No. comorbidities & $3.1 \pm 2.2$ \\
\hline \multicolumn{2}{|l|}{ Treatment profile } \\
\hline Prior use of csDMARD & $260(55)$ \\
\hline Prior use of bDMARD & $25(5)$ \\
\hline Current csDMARD & $425(91)$ \\
\hline Current no. csDMARD & $2.0 \pm 0.78$ \\
\hline Current bDMARD & $30(6)$ \\
\hline Subcutaneous & $24(5)$ \\
\hline Infusion & $6(1)$ \\
\hline Current oral corticosteroid use & $126(27)$ \\
\hline Current oral corticosteroid dose, $\mathrm{mg} / \mathrm{d}$ & $7.1 \pm 9.2$ \\
\hline
\end{tabular}

OBRI: Ontario Best Practices Research Initiative; SJC28: 28-joint swollen joint count; TJC28: 28-joint tender joint count; PtGA: patient's global assessment; PGA: physician's global assessment; CDAI: Clinical Disease Activity Index; HAQ-DI: Health Assessment Questionnaire-Disability Index; ESR: erythrocyte sedimentation rate; CRP: C-reactive protein; RF: rheumatoid factor; csDMARD: conventional synthetic disease-modifying antirheumatic drug; bDMARD: biologic DMARD.

significantly increased the risk of persistent depression over followup (OR 1.03, 95\% CI 1.01-1.05). Female sex (OR $2.68,95 \%$ CI $1.33-5.39)$ and greater number of comorbidities at baseline (OR 1.40, 95\% CI 1.24-1.59) were also positively associated with persistent depression (Table 2). Patient-reported outcomes including pain, fatigue, and disability were not associated with persistent depressive symptoms. A positive trend between categorical CDAI, defined as HDA, and persistent depression was also observed (adjusted OR 2.43, 95\% CI 1.00-5.99; Supplementary Table 2 , available with the online version of this article).

Association between CDAI components and persistent depression. To determine which CDAI core components may
Table 2. Association between baseline variables and persistent depression over the first 2 years of followup.

\begin{tabular}{lcc}
\hline Covariables & $\begin{array}{c}\text { Unadjusted OR } \\
(95 \% \text { CI })\end{array}$ & $\begin{array}{c}\text { Adjusted OR } \\
(95 \% \text { CI })\end{array}$ \\
\hline Continuous CDAI score & $1.03(1.01-1.04)$ & $\mathbf{1 . 0 3}(\mathbf{1 . 0 1 - 1 . 0 5})$ \\
Age & $1.00(0.99-1.02)$ & - \\
Female sex & $2.16(1.24-3.76)$ & $\mathbf{2 . 6 8}(\mathbf{1 . 3 3}-\mathbf{5 . 3 9})$ \\
Married status & $0.65(0.41-1.03)$ & - \\
White & $0.51(0.21-1.25)$ & - \\
Postgraduate education & $0.96(0.62-1.48)$ & - \\
High annual household income, & & \\
$\quad$ & & - \\
Private insurance & $0.93(0.57-1.52)$ & - \\
Rural residential area & $1.13(0.67-1.91)$ & - \\
Disease duration, yrs & $1.16(0.66-2.06)$ & - \\
Presence of erosions & $1.22(0.79-1.89)$ & - \\
ESR, mean & $0.76(0.48-1.20)$ & - \\
CRP, mean & $1.00(0.98-1.01)$ & - \\
HAQ-DI, mean & $1.00(0.99-1.01)$ & - \\
HAQ pain index, mean & $2.05(1.51-2.79)$ & $1.06(0.63-1.76)$ \\
Fatigue score, mean & $1.63(1.24-2.10)$ & $1.13(0.71-1.80)$ \\
No. comorbidities & $1.18(1.10-1.28)$ & $1.08(0.97-1.20)$ \\
RF-positive & $1.59(1.42-1.78)$ & $\mathbf{1 . 4 0}(\mathbf{1 . 2 4 - 1 . 5 9 )}$ \\
Prior use of csDMARD & $0.59(0.37-0.92)$ & $0.79(0.46-1.37)$ \\
Prior use of bDMARD & $0.52(0.33-0.81)$ & $\mathbf{0 . 5 5}(\mathbf{0 . 3 1 - 0 . 9 8})$ \\
Current use of bDMARD & $0.43(0.19-0.98)$ & $0.62(0.19-2.10)$ \\
Current use of csDMARD & $2.38(1.11-5.12)$ & $1.51(0.53-4.29)$ \\
Current oral glucocorticoid use & $1.38(0.62-3.08)$ & - \\
Oral glucocorticoid dose, mean, & $0.88(0.54-1.44)$ & - \\
$\quad$ & & - \\
mg/d & $1.00(0.96-1.04)$ & - \\
\hline & & \\
\hline
\end{tabular}

Values in bold face are statistically significant. CDAI: Clinical Disease Activity Index; ESR: erythrocyte sedimentation rate; CRP: C-reactive protein; HAQ-DI: Health Assessment Questionnaire-Disability Index; RF: rheumatoid factor; csDMARD: conventional synthetic disease-modifying antirheumatic drug; bDMARD: biologic DMARD.

be driving the association with disease activity and persistent depression, we conducted a multivariate regression analysis that was adjusted for explanatory covariates found to be significant in univariate analysis (sex, RF, prior use of csDMARD and bDMARD, current use of bDMARD, HAQ-DI, HAQ pain index, fatigue score, number of comorbidities). Higher SJC28 (OR 1.07, 95\% CI 1.01-1.12) and higher TJC28 (OR 1.14, 95\% CI 1.01-1.28) remained significant predictors of persistent depression, but not PGA or PtGA (Table 3).

Effect of sex on the association between disease activity and persistent depression. A significant interaction between sex and CDAI was observed in the multivariate analysis ( $\mathrm{p}=0.04$, data not shown). Therefore, the association between baseline CDAI and persistent depression was also examined separately for males and females (Supplementary Table 3, available with the online version of this article). There was no observed association between increased baseline CDAI and persistent depression in males. However, this association was significant in females (OR 1.04, 95\% CI 1.01-1.06), even after adjusting for potential confounders. In

Personal non-commercial use only. The Journal of Rheumatology Copyright $\odot$ 2018. All rights reserved. 
Table 3. Multivariable analysis evaluating the association between CDAI components at baseline and persistent depression, controlling for other significant baseline variables.

\begin{tabular}{|c|c|c|}
\hline $\begin{array}{l}\text { CDAI Components at } \\
\text { Enrolment }\end{array}$ & $\begin{array}{l}\text { Unadjusted OR } \\
\qquad(95 \% \mathrm{CI})\end{array}$ & $\begin{array}{l}\text { Adjusted OR } \\
\quad(95 \% \mathrm{CI})\end{array}$ \\
\hline SJC28 & $1.03(0.99-1.07)$ & $1.07(1.01-1.12)$ \\
\hline TJC28 & $1.06(1.03-1.09)$ & $1.14(1.01-1.28)$ \\
\hline PtGA & $1.19(1.09-1.31)$ & - \\
\hline PGA & $1.11(1.00-1.22)$ & - \\
\hline Female sex & $2.16(1.24-3.76)$ & $3.67(1.78-7.60)$ \\
\hline HAQ-DI & $2.05(1.51-2.79)$ & - \\
\hline HAQ pain index & $1.63(1.24-2.10)$ & - \\
\hline Fatigue score & $1.18(1.10-1.28)$ & - \\
\hline No. comorbidities & $1.59(1.42-1.78)$ & $1.41(1.25-1.60)$ \\
\hline RF-positive & $0.59(0.37-0.92)$ & - \\
\hline Prior use of csDMARD & $0.52(0.33-0.81)$ & $0.41(0.23-0.72)$ \\
\hline Prior use of bDMARD & $0.43(0.19-0.98)$ & - \\
\hline Current use of bDMARD & $2.38(1.11-5.12)$ & - \\
\hline Fatigue score & $1.18(1.10-1.28)$ & - \\
\hline
\end{tabular}

Values in bold face are statistically significant. CDAI: Clinical Disease Activity Index; SJC28: 28-joint swollen joint count; TJC28: 28-joint tender joint count; PtGA: patient's global assessment; PGA: physician's global assessment; HAQ-DI: Health Assessment Questionnaire-Disability Index; RF: rheumatoid factor; csDMARD: conventional synthetic disease-modifying antirheumatic drug; bDMARD: biologic DMARD. both males and females, a higher number of comorbidities was significantly associated with increased odds of persistent depression, whereas prior use of csDMARD was associated with lower odds of persistent depression (Supplementary Table 3).

Association between CDAI and risk of depression at any time over 2 years of followup. Using a GEE model, there was a trend between increased CDAI over time and risk of depression in univariate analysis (OR 1.01, 95\% CI 1.00-1.01; Table 4). After adjusting for potential confounders, the association remained marginally significant (OR 1.01, 95\% CI 1.00-1.01). In multivariate analysis, categorical CDAI was significantly associated with depression, and both MDA (OR 1.14, 95\% CI 1.04-1.26) and HDA (OR 1.26, 95\% CI 1.12-1.42) increased self-reported depression compared to patients in remission or LDA. This association was also significant when patients were dichotomized into MDA or HDA status (OR 1.22, 95\% CI 1.10-1.35) compared to patients in remission/low disease activity status (Table 4, Model 2). In this analysis, female sex, greater number of baseline comorbidities, higher fatigue score over time, and use of a subcutaneous bDMARD were also significantly associated with increased odds of depression over 2 years (Table 4).

Association between CDAI and self-reported treatment for depression (sensitivity analysis). We conducted a sensitivity

Table 4. Association between CDAI change over time and self-reported depression over 2 years of followup using general estimating equation.

\begin{tabular}{|c|c|c|c|}
\hline Variables & $\begin{array}{l}\text { Unadjusted OR } \\
\quad(95 \% \mathrm{CI})\end{array}$ & $\begin{array}{c}\text { Adjusted OR } \\
(95 \% \text { CI }) \\
\text { Model 1* }\end{array}$ & $\begin{array}{c}\text { Adjusted OR } \\
(95 \% \text { CI }) \\
\text { Model 2** }\end{array}$ \\
\hline CDAI over time & $1.01(1.00-1.01)$ & $1.01(1.00-1.01)$ & - \\
\hline \multicolumn{4}{|l|}{ CDAI category over time } \\
\hline Remission or LDA & Ref & - & Ref \\
\hline MDA & $1.14(1.06-1.22)$ & - & $1.14(1.04-1.26)$ \\
\hline HDA & $1.26(1.13-1.41)$ & - & $1.26(1.12-1.42)$ \\
\hline \multicolumn{4}{|l|}{ CDAI binary over time } \\
\hline Remission or LDA & Ref & - & Ref \\
\hline MDA or HDA & $1.18(1.10-1.27)$ & - & $1.22(1.10-1.35)$ \\
\hline \multicolumn{4}{|l|}{ Covariates } \\
\hline Female sex & $1.88(1.15-3.08)$ & $1.93(1.15-3.25)$ & - \\
\hline No. comorbidities & $1.53(1.39-1.70)$ & $1.32(1.19-1.47)$ & - \\
\hline Positive RF & $0.60(0.39-0.92)$ & $0.79(0.50-1.26)$ & - \\
\hline Fatigue score $(0-10)$ & $1.19(1.11-1.28)$ & $1.14(1.05-1.24)$ & \\
\hline Prior use of csDMARD & $0.52(0.34-0.80)$ & $0.74(0.46-1.19)$ & \\
\hline Prior use of bDMARD & $0.43(0.19-0.95)$ & $0.53(0.19-1.49)$ & - \\
\hline Current use and route of bDMARD & & & - \\
\hline Infusion & $1.01(1.00-1.03)$ & $0.95(0.76-1.19)$ & - \\
\hline Subcutaneous & $1.10(0.97-1.25)$ & $1.31(1.06-1.63)$ & \\
\hline
\end{tabular}

Values in bold face are statistically significant. * Model 1: adjusted for sex, positive RF, prior use of csDMARD and bDMARD, current bDMARD administration route, total number of bDMARD, number of comorbidities, and fatigue score. ** Model 2: CDAI category and binary variables adjusted for the relevant covariates (OR for these covariates are not shown in the table). CDAI: clinical disease activity index; LDA: low disease activity; MDA: medium disease activity; HDA: high disease activity; RF: rheumatoid factor; csDMARD: conventional synthetic disease-modifying antirheumatic drug; bDMARD: biologic DMARD.

Personal non-commercial use only. The Journal of Rheumatology Copyright (C) 2018. All rights reserved. 
analysis using self-reported treatment for depression as an alternative outcome definition. An association between higher baseline CDAI and risk of persistent depression using this outcome was found and was similar in magnitude to the primary analysis (OR 1.03, 95\% CI 1.01-1.06; data not shown).

\section{DISCUSSION}

In this large sample of patients with ERA managed in routine clinical practice, self-reported depression was highly prevalent at baseline (26\%). Despite the frequent episodic features of mood disorders, persistent depression was reported in $90 \%$ of subjects and was associated with HDA at baseline. The effect of high CDAI and persistent mood symptoms was particularly evident among females.

Depression and RA studies have predominantly evaluated the negative effect of depression on RA treatment response. Our results add to the literature, suggesting that a bidirectional relationship between mood symptoms and disease can exist $^{13}$. Our results validate our hypothesis that initial HDA affects concurrent depressive symptoms but has prognostic value for persistent depression. These findings are important because there are limited reports confirming this in a strictly ERA population. It may be additional support to treat ERA to a low disease state, or preferably remission, for the potential to avoid the development of chronic comorbidity $9,11,12,14,15$. Our results also indicate that changes in CDAI over time, particularly to moderate or high disease states, increases the risk of depression over the first 2 years of followup. This suggests that the identification and management of depressive symptoms should occur as soon as possible. Future studies are needed to clarify whether depression can be modified or prevented by aggressive disease control during the early treatment "window of opportunity".

The prevalence of self-reported depressive symptoms in our study of $26 \%$ is lower than reports in other ERA studies, with baseline prevalence ranging from $35 \%$ to $47 \%$ and that likely relates to the heterogeneous outcome measures used to define depression ${ }^{11,12,15}$. We show that persistent self-reported depression was the common pattern $(90 \%$ reporting initial depression remained depressed). Stable rates of depression have also been verified by Smedstad, et al, while another study found that ERA subjects actually became more depressed longitudinally ${ }^{16,17}$. This contrasts with other reports of a decline in psychological distress over time in ERA subjects, particularly beyond the third year of disease $11,12,15,18$. Our results may have differed if we extended the period of followup, since it has been suggested that emotional adaptation and coping mechanisms that influence mood may take several years and only become apparent once disease is more established ${ }^{15}$.

To our knowledge, our study is the first to investigate which components of CDAI may be most predictive of persistent depression in ERA. It is well established that mood symptoms, even in early disease, lead to higher patient global scores ${ }^{19,20}$. Given the subjectivity of mood symptoms, we anticipated high baseline PtGA to be strongly associated with persistent depression. Interestingly, PtGA was not a significant mediator of the CDAI and persistent depression relationship. Further, patient experiences frequently encompassed in the PtGA score (pain, fatigue, disability) were also not significant in our analyses and contradicts findings that HAQ disability and pain are significant predictors of future mood disorders in most other ERA studies ${ }^{12,15,16}$. The only core components associated with persistent depression in our study were TJC and SJC. This implies that synovitis may indeed have negative consequences beyond the physical manifestations of RA. In keeping with this, previous csDMARD use was protective against persistent depressive symptoms, suggesting that early treatment may have an effect on subsequent comorbidity. Bacconnier, et al also found that higher TJC predicted psychological distress among ERA subjects at Month 12, but in contrast to our results, no treatment regimen was associated with depression or anxiety at any timepoint over 3 years ${ }^{12}$. Other studies have indicated that changes in psychological distress are not predicted by disease-related variables or biological markers such as ESR values $^{12,14,17}$. Although not required to calculate our disease activity measure of interest (CDAI), we also did not find objective measures of systemic inflammation (ESR or CRP) to predict persistent depression in our models.

Our study demonstrated that female sex was independently associated with baseline and persistent depression. This is in keeping with analyses that female sex is a general population risk factor for depression and more severe depression, which has also been replicated in studies of RA $^{6,18,21,22}$. However, we are not aware of other ERA cohorts analyzing the effect modification of sex on disease activity and persistent depression. We found that the relationship between increasing CDAI and persistent depressive symptoms was significantly modified by female but not male sex. Higher baseline comorbidity profile was positively associated with depression in both sexes. Similar to our primary analysis, prior use of csDMARD (but not current DMARD or biologic use) reduced the risk of persistent depression. There are many postulated reasons for this differential risk, including true biological and immunological differences. However, the small sample size of males in our study limits us to draw firm conclusions. We also cannot discern the effect of stress, gender roles, illness perception, coping, or emotional expression that are known to affect mood symptoms and may differ by $\operatorname{sex}^{23}$. For now, these findings may alert clinicians to screen for depressive symptoms, particularly among women with initial HDA and in the presence of other health conditions. This subgroup appears to have a multiplicative risk for persistent depression and will warrant further investigation.

Personal non-commercial use only. The Journal of Rheumatology Copyright $\odot$ 2018. All rights reserved 
The major strength of our study was its longitudinal design and large sample of subjects with ERA who are representative of real-world clinical practice. To our knowledge, we are among the first to attempt to look at persistent depression, although a unified definition is needed to allow for cross-study comparisons. Our well-described cohort allowed us to control for a large number of variables, including sociodemographic, objective clinical variables, patient-reported outcomes, and treatments as possible confounders of the relationship between disease activity and persistent depression. However, some limitations include the lack of any specific diagnostic criteria for depression or measure of depression severity. We opted to use a 1-item question for self-reported depression because standard composite screening tools for depression were not available at the time of data collection. However, no single depression instrument has been validated for use in RA and several tools measure psychological symptoms more broadly (suffering, anxiety, etc.), whereas the focus of our research question was depressive symptoms. We took care to conduct a sensitivity analysis, changing the outcome to "self-reported treatment for depression" and this confirmed the certainty of our main findings. Another limitation is that we were not able to examine the role of social support, coping, sleep, and a number lifestyle factors or specific comorbid conditions (e.g., fibromyalgia) that may have confounded our results. We were also not able to assess intervisit depressive symptomatology to determine if the prevalence of persistent depression remains stable or is indeed more episodic over time. Finally, given the observational characteristics of our study, even though the association between HDA and symptomatic depression was assessed, no causal inference can be made.

Our study demonstrates that initial HDA, even in early disease, may set the stage for persistent depressive symptoms. This risk seems particularly modified in females with active disease and among those with coexisting comorbidity and represents an area for targeted focus, screening, and further research. Our findings suggest that controlling disease activity over time to the lowest possible state may have the added benefit of limiting psychiatric comorbidity. Future studies on the optimal evaluation and treatment of depression, and how this may coincide or complement the management of patients with ERA, is needed.

\section{ACKNOWLEDGMENT}

We would like to thank all the Ontario Best Practices Research Initiative (OBRI) participants and OBRI investigators for contributing their data.

\section{ONLINE SUPPLEMENT}

Supplementary material accompanies the online version of this article.

\section{REFERENCES}

1. Radner H, Yoshida K, Smolen JS, Solomon DH. Multimorbidity and rheumatic conditions-enhancing the concept of comorbidity. Nat Rev Rheumatol 2014;10:252-6.

2. Matcham F, Rayner L, Steer S, Hotopf M. The prevalence of depression in rheumatoid arthritis: a systematic review and meta-analysis. Rheumatology 2013;52:2136-48.

3. Dougados M, Soubrier M, Antunez A, Balint P, Balsa A, Buch MH, et al. Prevalence of comorbidities in rheumatoid arthritis and evaluation of their monitoring: results of an international, cross-sectional study (COMORA). Ann Rheum Dis 2014;73:62-8.

4. Margaretten M, Julian L, Katz P, Yelin E. Depression in patients with rheumatoid arthritis: description, causes and mechanisms. Int J Clin Rheumtol 2011;6:617-23.

5. Ang DC, Choi H, Kroenke K, Wolfe F. Comorbid depression is an independent risk factor for mortality in patients with rheumatoid arthritis. J Rheumatol 2005;32:1013-9.

6. Jacob L, Rockel T, Kostev K. Depression risk in patients with rheumatoid arthritis in the United Kingdom. Rheumatol Ther 2017;4:195-200.

7. Wang SL, Chang CH, Hu LY, Tsai SJ, Yang AC, You ZH. Risk of developing depressive disorders following rheumatoid arthritis: a nationwide population-based study. PLoS One 2014;9:e107791.

8. Dantzer R, O'Connor JC, Freund GG, Johnson RW, Kelley KW. From inflammation to sickness and depression: when the immune system subjugates the brain. Nat Rev Neurosci 2008;9:46-56.

9. Kekow J, Moots R, Khandker R, Melin J, Freundlich B, Singh A. Improvements in patient-reported outcomes, symptoms of depression and anxiety, and their association with clinical remission among patients with moderate-to-severe active early rheumatoid arthritis. Rheumatology 2011;50:401-9.

10. Jamshidi AR, Banihashemi AT, Paragomi P, Hasanzadeh M, Barghamdi M, Ghoroghi S. Anxiety and depression in rheumatoid arthritis: an epidemiologic survey and investigation of clinical correlates in Iranian population. Rheumatol Int 2016;36:1119-25.

11. Overman CL, Bossema ER, van Middendorp H, Wijngaards-de Meij L, Verstappen SM, Bulder M, et al. The prospective association between psychological distress and disease activity in rheumatoid arthritis: a multilevel regression analysis. Ann Rheum Dis 2012;71:192-7.

12. Bacconnier L, Rincheval N, Flipo RM, Goupille P, Daures JP, Boulenger JP, et al. Psychological distress over time in early rheumatoid arthritis: results from a longitudinal study in an early arthritis cohort. Rheumatology 2015;54:520-7.

13. Rathbun AM, Reed GW, Harrold LR. The temporal relationship between depression and rheumatoid arthritis disease activity, treatment persistence and response: a systematic review. Rheumatology 2013;52:1785-94.

14. Heimans L, Wevers-de Boer KV, Visser K, Ronday HK, Steup-Beekman GM, van Oosterhout M, et al. The relationship between disease activity and depressive symptoms severity and optimism - results from the IMPROVED study. Clin Rheumatol 2013;32:1751-7.

15. Persson LO, Larsson BM, Nived K, Eberhardt K. The development of emotional distress in 158 patients with recently diagnosed rheumatoid arthritis: a prospective 5-year follow-up study. Scand J Rheumatol 2005;34:191-7.

16. Smedstad LM, Vaglum P, Moum T, Kvien TK. The relationship between psychological distress and traditional clinical variables: a 2 year prospective study of 216 patients with early rheumatoid arthritis. Br J Rheumatol 1997;36:1304-11.

17. Sharpe L, Sensky T, Allard S. The course of depression in recent onset rheumatoid arthritis: the predictive role of disability, illness perceptions, pain and coping. J Psychosom Res 2001;51:713-9.

18. Evers AW, Kraaimaat FW, Geenen R, Jacobs JW, Bijlsma JW. Longterm predictors of anxiety and depressed mood in early rheumatoid arthritis: a 3 and 5 year followup. J Rheumatol 2002:29:2327-36

19. Leblanc-Trudeau C, Dobkin PL, Carrier N, Cossette P, de Brum-Fernandes AJ, Liang P, et al. Depressive symptoms predict

Personal non-commercial use only. The Journal of Rheumatology Copyright @ 2018. All rights reserved. 
future simple disease activity index scores and simple disease activity index remission in a prospective cohort of patients with early inflammatory polyarthritis. Rheumatology 2015;54:2205-14.

20. Euesden J, Matcham F, Hotopf M, Steer S, Cope AP, Lewis CM, et al. The relationship between mental health, disease severity, and genetic risk for depression in early rheumatoid arthritis. Psychosom Med 2017;79:638-45.

21. Crotty M, McFarlane AC, Brooks PM, Hopper JL, Bieri D, Taylor SJ. The psychosocial and clinical status of younger women with early rheumatoid arthritis: a longitudinal study with frequent measures. Br J Rheumatol 1994;33:754-60.
22. Ramjeet J, Koutantji M, Barrett EM, Scott DG. Coping and psychological adjustment in recent-onset inflammatory polyarthritis: the role of gender and age. Rheumatology 2005;44:1166-8.

23. Tillmann T, Krishnadas R, Cavanagh J, Petrides KV. Possible rheumatoid arthritis subtypes in terms of rheumatoid factor, depression, diagnostic delay and emotional expression: an exploratory case-control study. Arthritis Res Ther 2013;15:R45. 